\title{
It ain't necessarily so: Gravitational Waves and Energy Transport
}

“Weia! Waga! Woge, du Welle! Walle zur Wiege! Wagalaweia! Wallala weiala weia!”

Richard Wagner: "Das Rheingold”, Scene 1

- Patrick M. Dürr, patrick.duerr@oriel.ox.ac.uk, Oriel College, University of Oxford, UK -

\begin{abstract}
:
In the following paper, I review and critically assess the four standard routes commonly taken to establish that gravitational waves (GWs) possess energy-momentum: the increase in kinetic energy a GW confers on a ring of test particles, Bondi/Feynman's Sticky Bead Argument of a GW heating up a detector, nonlinearities within perturbation theory, taken to reflect the fact that gravity contributes to its own source, and the Noether Theorems, linking symmetries and conserved quantities. Each argument is found to either to presuppose controversial assumptions or to be outright spurious. I finally examine the standard interpretation of binary systems, according to which orbital decay is explained in terms of the system's energy being via GW energymomentum transport. I contend that a better interpretation, drawing only on the general-relativistic Equations of Motions and the Einstein Equations, is available - and in fact preferable; thereby also an inference to the best explanation for the vindication of GW energy-momentum is blocked.
\end{abstract}

Key words: General Relativity, Gravitational Waves, Sticky Bead, binary systems, Problem of Motion

\section{Content}

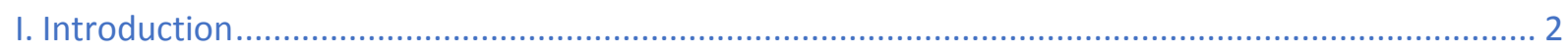

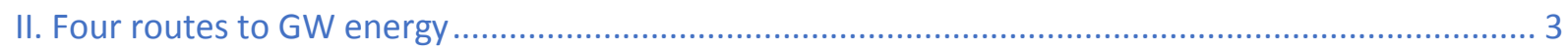

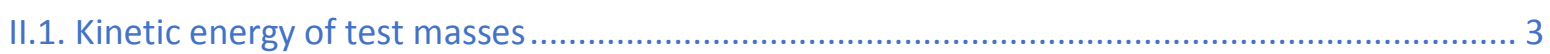

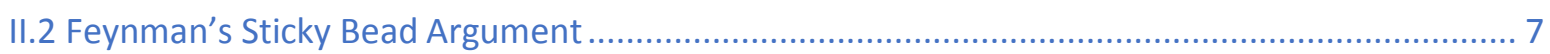

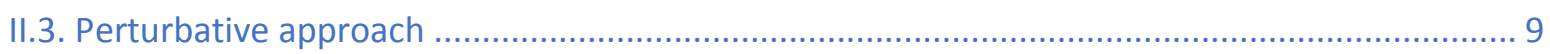

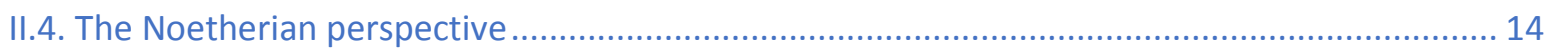

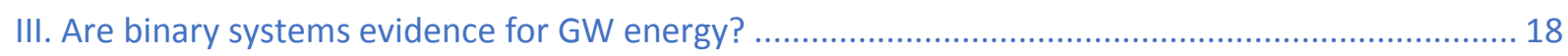

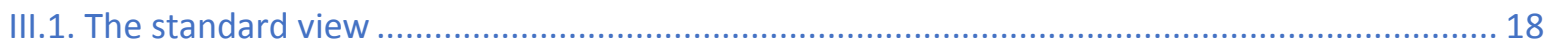

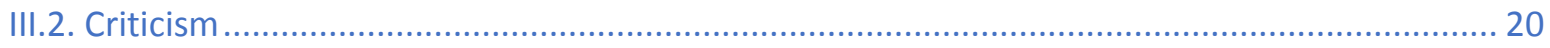

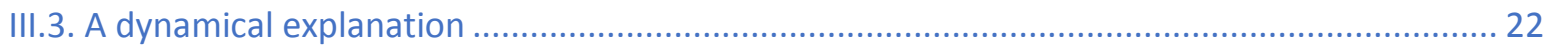

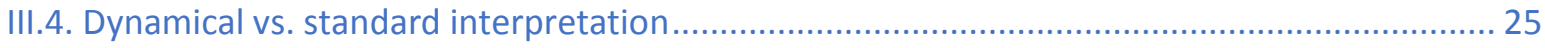




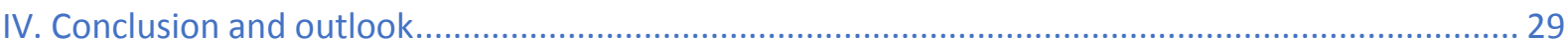

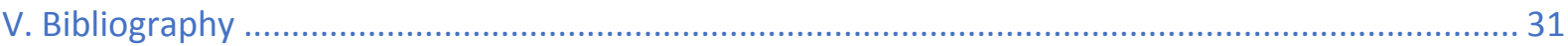

\section{Introduction}

In the following I endeavor to examine whether GWs carry energy-momentum.

Many will deem this an old hat. A monograph on GW astrophysics exemplarily proclaims: "Since gravitational waves produce a real physical effect [...] - it is clear that the wave must be carrying energy." ${ }^{1}$ Such an effect is the orbital spin-up of double pulsars, discovered by Hulse and Taylor in 1974: On its standard interpretation, the double pulsars demonstrate the binary system's loss of energy by radiating away energy via GWs; as a result, the pulsars move closer to each other - a prediction corroborated with amazing accuracy. ${ }^{2}$

At the latest since the repeated direct detection of GWs the existence of GWs is beyond reasonable dispute. ${ }^{3}$ But perhaps the standard interpretation of their effects as depleting the binaries' orbital energy deserves a second glance:

- Petkov has recently stressed that the pulsars are in free-fall and hence move inertially. Shouldn't therefore their kinematic state remain unaltered? In particular, shouldn't the binaries preserve their energy? ${ }^{4}$

- More generally, as Norton remarked: If, in GR, the gravitational force is "geometrised away" shouldn't this compromise the very notion of gravitational energy, as well? ${ }^{5}$

- Closely related is the issue of energy-momentum conservation. As we know from Analytic Mechanics, conservation of energy and momentum is tied up with the symmetries of space and time. ${ }^{6}$ But isn't space-time, according to GR, warped, generically lacking any symmetries? Shouldn't this affect the validity of conservation laws?

Following Einstein's advice never to stop questioning, I shall subsequently challenge the received ascription of energy-momentum to GW by critically examining the four standard arguments taken to establish the energy-momentum of GWs. The first two adduce phenomenological effects GWs produce: The "Argument from Kinetic Energy" consists in the change in kinetic energy of a ring of free particles (sect. II.1) hit by a GW; the other effect consists in the heating-up of a detector whose constituents are held together by internal forces (sect. II.2). Of a more systematic nature are two other arguments, the natural energymomentum of a GW, obtained from a decomposition of the metric into a background and perturbations, and an application of Noether's Theorem (II.4), respectively. All four arguments are found to be deficient - at least, when espousing GR's "geometric" interpretation,

\footnotetext{
${ }^{1}$ Anderson/Creighton (2011), p. 66

2 E.g. Will (2014), sect. 6,7

${ }^{3}$ Cf. Castelvecchi/Witze (2016)

${ }^{4}$ Cf. Petkov (2012), Appendix C

${ }^{5}$ Norton (2012), sect. 3.9

${ }^{6}$ E.g. Landau/Lifshitz (1976) §6
} 
according to which gravitational effects are re-conceptualised as manifestations of a nonMinkowskian chronogeometric and inertial structure.

Below, I adopt this geometric interpretation of GR throughout. This is not to deny the virtues (let alone, existence) of alternatives, esp. the "particle physicist view" (Pitts), which conceives of the metric as a physical field. ${ }^{7}$ Apart from the fact that it is the philosophers' professional duty to explicate presuppositions, a conceptually rigorous development of the geometric interpretation, to which I hope my analysis will contribute, is likely to facilitate a systematic adjudication between it and rivalling choices. ${ }^{8}$

If the standard arguments for ascribing GWs energy-momentum fail, how then to account for their paradigmatic effects? Section III adverts to the standard treatment of binary systems (III.1-2), and sketches how their spurious explanation in terms of the energy GWs carry can be replaced by a more satisfactory "dynamical" one (III.3), which appeals solely to the generalrelativistic Equations of Motion and the Einstein Equations. Thereby, also an inference to the best explanation, intended to establish GW energy-momentum is blocked (III.4). I summarise the results of my analysis in sect. IV and outline two promising lines of further inquiry.

\section{Four routes to GW energy}

In this section, I revisit and evaluate the four arguments canonically cited for ascribing GWs energy-momentum:

(1) A GW incident on free particles, initially at rest in a lab frame, sets them into motion.

(2) A GW can induce heat in a detector.

(3) Within perturbation theory, higher-order contributions can be naturally construed as the GWs' energy-momentum.

(4) The framework of the Noether Theorems leads to energy-momentum in the same way as other field theories.

I submit, none is compelling, either because the argument rests on conceptual distinctions not available within a geometrically interpreted GR (as is the case with (1) and (3)) or (as is the case with (2) and (4)) because they hinge on implicit, tenuous assumptions that require -at the least- substantive additional arguments.

\section{II.1. Kinetic energy of test masses}

The default argument for the energy of GWs turns on the kinetic effects of a GW on test particles, otherwise at rest; this energy is supposed to be extracted from the GW.

\footnotetext{
${ }^{7}$ Cf. Pitts (2016a,b)

${ }^{8}$ Cf. Lehmkuhl (2008); Rey (2013)
} 
The thought experiment rests on a perturbative treatment of GR, so-called linear GW theory 9 . (The perturbative approach will be studied in full generality in II.3.) Within linearised theory, one assumes that the gravitational field is weak, such that the spacetime metric $g_{a b}$ deviates only slightly from a flat Minkowski background $\left(\eta_{a b}\right):^{10}$

$$
g_{a b}=\eta_{a b}+h_{a b}, \text { where }\left|h_{a b}\right| \ll 1 .
$$

In this perturbative order $h_{a b}$ can be treated like a symmetric tensor field under global Lorentz transformations. (From now on Greek indices will denote approximately Lorentz tensors; indices thus are also raised/lowered w.r.t. the Minkowski metric.) Linearised theory thus effectively is a special-relativistic theory of gravity for weak fields.

What happens then to the General Covariance of general-relativistic equations? The invariance of the latter under infinitesimal coordinate transformations, $x^{\mu} \rightarrow x^{\prime \mu}=x^{\mu}+$ $\xi^{\mu}(x)$ (where $\xi^{\mu}(x)$ is an arbitrary function of the same order of smallness as $h_{a b}$ ), in linearised theory becomes invariance under gauge transformations of the form $h_{\mu \nu} \rightarrow h_{\mu \nu}^{\prime}=$ $h_{\mu \nu}-2 \partial_{(\mu} \xi_{v)}$.

Expanding the general-relativistic tensors in powers of the perturbation $h_{\mu \nu}$ yields in leading order the corresponding quantities in linearised theory (denoted by the scripted symbols), such as the linearised Einstein tensor:

$$
\mathcal{G}_{\mu \nu}=\partial_{\mu, \nu}^{2} h+\square h_{\mu \nu}-\partial_{\lambda, \nu}^{2} h_{\mu}^{\lambda}-\left(\square h-\partial_{\kappa, \lambda}^{2} h^{\kappa \lambda}\right) \eta_{\mu \nu}
$$

with $h:=\eta^{\mu \nu} h_{\mu \nu}$ and the flat spacetime d'Alembertian $\square:=\eta^{\mu v} \partial_{\mu, v}^{2}$. For consistency, the energy-momentum tensor $T_{\mu \nu}$ on the r.h.s. of the Einstein Equations must likewise be of first order in the perturbations $\left(T_{\mu \nu} \approx \mathcal{T}_{\mu \nu}\right)$.

Harnessing the gauge freedom for the gravitational field, the linearised Einstein Equations, $\mathcal{G}_{\mu \nu}=-\frac{16 \pi G}{c^{4}} \mathcal{T}_{\mu \nu}$, simplify for a particular gauge (the so-called "TT-gauge"), to an inhomogeneous wave equation. Its general solution is obtained via Greens functions. For the purposes of GWs, we may restrict ourselves to the vacuum case $\left(\mathcal{T}_{\mu \nu}=0\right)$ with plane wave packets as solutions, $h_{\mu \nu}=\int d^{3} \vec{k} A_{\mu \nu}(\vec{k}) e^{i k_{\lambda} x^{\lambda}}$ (with a generic, wave-vector dependent function $A_{\mu \nu}$, the so-called polarization tensor). For concrete astrophysical applications, such wave packets and their effects are further evaluated.

One may demur at imposing the above TT-gauge condition. However, in the context of the cosmological perturbation formalism this can be shown not to curtail the general validity of the argument: Only the transverse, traceless degrees of freedom of the metric genuinely (i.e. not as an illusory artefact of a coordinate choice) obey a wave-like equation (with the other components satisfying an equation of the Poisson type) and hence can be identified as radiative. ${ }^{11}$

\footnotetext{
${ }^{9}$ E.g. Misner et al. (1973), Ch. 35; Hobson et al. (2006), Ch. 17

${ }^{10}$ For GWs incident on our GW detectors $h_{\mu \nu}$ is typically of order $10^{-21}$. For comparison, the absolute values of gravitational fields in our solar system are still quite small, typically: $\left|h_{i j}\right| \lesssim 10^{-6}$, cf. Misner et al. (1973), Ch. 39. ${ }^{11} \mathrm{Cf}$. Flanagan/Hughes (2005). Such a decomposition of the metric into radiative TT-pieces, (unphysical) gauge pieces and gravitational pieces is always possible and unique.
} 
Like an electromagnetic one, a GW possesses two linear (or two circular) polarizations. Their respective designations, $\oplus$ and $\otimes$, indicate the axes along which a ring of a test particles is distorted. The effect a purely $\oplus$-polarised GW travelling along the z-axis, $h_{a b}^{\oplus}=$ $\cos k(c t-z) e_{a b}^{\oplus}$ produces in a transverse circle of particles is shown in Figure 1.

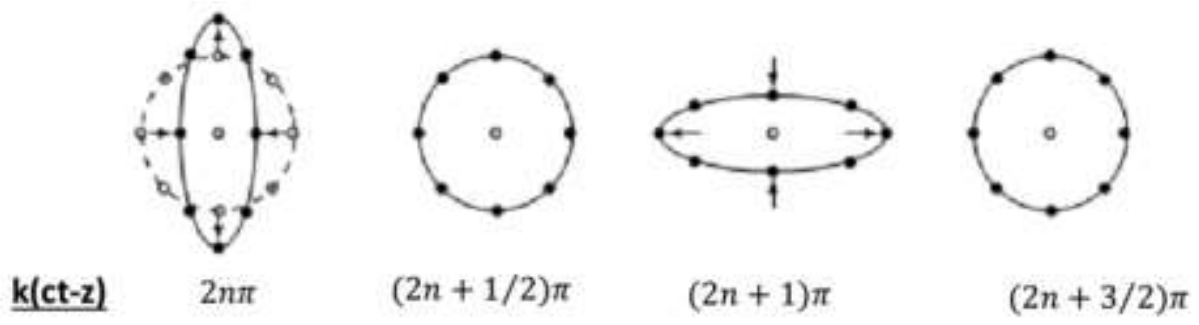

Fig. $1^{12}$ : Deformations of a ring of test particles in the $x-y$ plane. The initial configuration is shown by the open dots.

For laboratory practices, it is apposite to adopt the so-called proper detector frame $\mathrm{e}^{13}-\mathrm{a}$ coordinate system in which one fixes the origin, and then uses rigid rulers to delineate coordinates, enforcing thus the geometry measured in these coordinates to be Euclidean.

The GW incident on our above ring of test particles (each assumed to be of mass $m$ ) deforms the latter, stretching and squeezing the ring in $\mathrm{x}$ - and $\mathrm{y}$-direction, respectively: $\delta x(t)=$ $\frac{h_{\oplus}}{2} \sin \omega t$ and $\delta y(t)=-\frac{h_{\oplus}}{2} \sin \omega t$. (Fig.1 depicts the deformations by the black arrows). The particles start moving, thereby changing their kinetic energy:

$$
E_{k i n}=\frac{m}{2}\left(\delta \dot{x}^{2}+\delta \dot{y}^{2}\right)=\frac{m}{4} h_{\oplus}^{2} \omega^{2} \cos ^{2} \omega t .
$$

Whence this energy gain - so the rationale of the first canonical argument goes - if not from the GW?

What to make of this Argument from Kinetic Energy? Two objections can be made: The first one repudiates the argument for its appeal to a non-inertial frame; the second objection targets the ambiguity of "kinetic energy" in a GR context.

At the core of the Argument from Kinetic Energy lies its reliance on the proper detector frame. However, this reference is not physically privileged in GR. In fact, with the coordinate proper detector frame's coordinates being delineated by rigid rulers, according to a theorem first formulated by Helmholtz ${ }^{14}$, a proper detector frame cannot be physically realized in space of variable curvature, unless forces are operant that counterbalance the deformations the curvature inflicts on extended bodies, such as rigid rulers.

More natural ${ }^{15}$ and privileged - as inertial - is the so-called TT-frame: In it coordinate labels are attached to freely falling test particles. Lest the picture be unnecessarily obfuscated by inertial

\footnotetext{
12 Taken from Hobson et al. (2006), p. 506.

${ }^{13}$ E.g. Maggiore (2008), Ch. 1.3.3

${ }^{14}$ Cf. Mittelstaedt (1981), Ch. II §3.

The first rigorous proof seems to stem from Sophus Lie in 1888. Thanks to Marco Giovanelli for this hint.

${ }^{15}$ Kennefick (2007), p. 131, nicely likens the TT-frame with the natural way oceanographers would introduce coordinates for orientation on sea.
} 
effects, such as the fictitious forces, known from Classical Mechanics, let us re-evaluate the above situation of test masses in these inertial reference frames.

In the TT frame, however, as follows immediately from the geodesic equation, no particle changes its coordinate position, $\frac{d x^{a}}{d \tau}=0$. Hence, the GW does not affect the particles' kinetic energy.

The non-gain of kinetic energy in the TT frame notwithstanding, the physical (spatial) distances/separation, $\sqrt{\left|g_{i j} \xi^{i} \xi^{j}\right|}$, for a spacelike vector $\xi=\left(0, \xi^{i}\right)$, do, of course, change while the coordinate distance $\sqrt{\left|\delta_{i j} \xi^{i} \xi^{j}\right|}$ remains constant. (To be sure, one can recover the rate of change of the physical distances ${ }^{16}$ (i.e. and interpret the result as a force law, coinciding with the proper detector frame description). However, within the spacetime setting inherent in Minkowskian physics, co-moving coordinate and physical (proper) distances coincide.)

This objection to the Kinetic Argument's privileged treatment of a non-inertial reference frame employed the notion of kinetic energy - tacitly assuming that within GR, this is an uncontroversial notion. A different line of attack therefore arises from disputing this.

First, recall that in Classical Mechanics, kinetic energy plays two distinct roles, only contingently related: On the one hand it is the residual part of the total energy, after subtracting the contributions from all dynamical interactions; on the other hand, kinetic energy is the numerical value of the Lagrangian that yields the equation of motion of free particles. ${ }^{17}$

In the transition to general-relativistic mechanics, these two roles are no longer played by the same object. An evaluation of kinetic energy is not as straightforward as my preceding thoughts may have insinuated.

To remedy this, consider - in a manner familiar from SR ${ }^{18}$ - a particle's energy (with mass $m$ and the 4-momentum $p^{a}$ ) $E[\xi]=g_{a b} p^{a} \xi^{b}$, relative to an observer with the 4-velocity $\xi^{a}$. Subtract from this energy $E[\xi]$ the particle's rest energy

$$
E_{\mathrm{kin}}=p_{a} \xi^{a}-m c^{2} \text {. }
$$

We can evaluate this further via the length of the norm of the observer's 4-velocity, $c^{2}=$ $g_{a b} u^{a} u^{b}=c^{2} g_{00}\left(\frac{d t}{d \tau}\right)^{2}+2 c g_{0 i} v^{i} \frac{d t}{d \tau}+c^{2} g_{i j} v^{i} v^{j}\left(\frac{d t}{d \tau}\right)^{2}$, with $\tau$ denoting the affine parameter and $v^{j}=\frac{d x^{j}}{d t}$ the coordinate velocity, respectively.

\footnotetext{
${ }^{16}$ E.g. Ohanian/Ruffini (2013), Ch. 5.2.

${ }^{17}$ This is how kinetic energy is occasionally even defined, cf. Landau/Lifshitz (1976), §4.

${ }^{18}$ E.g. Malament (2012), Ch. 2.4
} 
For a GW in TT-gauge (such that $g_{0 i}=0$ ) and an inertial observer, hence $\xi^{i}=0$, one obtains: $\xi^{0}=c \frac{d t_{(\mathrm{obs})}}{d \tau}=\frac{c}{\sqrt{g_{00}}}$ and hence for the kinetic energy: ${ }^{19}$

$$
E_{k i n}=m c^{2}\left(\sqrt{\frac{g_{00}}{g_{00}+g_{i j} v^{i} v^{j}}}-1\right)
$$

We are now in a position to evaluate this general-relativistic analogue of kinetic energy: With the inertial coordinates of the TT frame, in which the particle positions don't change, $v^{j}=0$, the kinetic energy is indeed zero. Our previous counterargument to the Argument from Kinetic Energy thus goes through.

But does this also hold for the correct GR counterpart to the second notion of kinetic energy? The GR Lagrangian whose variation yields the equation of motion for a free massive particle, in generalisation of the special-relativistic case, reads:

$$
\mathcal{L}_{0}=\sqrt{\left|g_{a b} \frac{d x^{a}}{d \tau} \frac{d x^{b}}{d \tau}\right|}
$$

Being a scalar with the constant numerical value $c$ for massive particles, and zero for photons, it does not change, even when a GW passes through the particle. Consequently, again our counterargument goes through.

To summarise: The Argument from Kinetic Energy draws its force from allocating non-inertial frames a special status that from a GR perspective is unjustified. A description of the phenomena in GR's inertial frames the apparent increase of kinetic energy lapses.

A related phenomenological argument is the Sticky Beads Argument. Rather than free particles, the Sticky Beads Argument involves systems with friction.

\section{II.2 Bondi-Feynman's Sticky Bead Argument}

The idea is similar to the Argument from Kinetic Energy: The thought experiment assumes that a GW can heat up matter, the thermal energy allegedly being extracted from the GW energy.

In the 1950s, during an era where the emission of GWs was still fiercely debated ${ }^{20}$, Bondi and Feynman (it seems, independently) proposed a simple, qualitative thought experiment ${ }^{21}$ that appears to compellingly demonstrate that GWs must carry energy (and hence must be real). ${ }^{22}$

\footnotetext{
19 To reassure the reader that we are on the right track: In Minkowski spacetime, this reduces to the familiar special-relativistic expression for kinetic energy $m c^{2}\left(\frac{1}{\sqrt{1-v^{2} / c^{2}}}-1\right)=\frac{1}{2} m v^{2}+\frac{3}{8} m \frac{v^{4}}{c^{2}}+\cdots$.

${ }^{20}$ Cf. Kennefick (2007), Ch. 5-7

${ }^{21}$ Cf. Feynman et al. (2002), Foreword.

${ }^{22}$ It is worth pointing out that many, explicitly e.g. Bunge (2017), seem to regard energy transport of GWs also as a necessary criterion for their existence. The view I am advocating here is that, while I do not deny that energy
} 


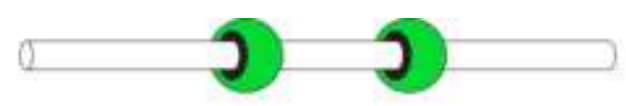

Fig. 2:

Upper part: sticky beads at rest Lower part: The incident GW sets the beads in motion along the stick, which causes friction. The stick heats up (yellow).

(picture taken from Larson (2015))

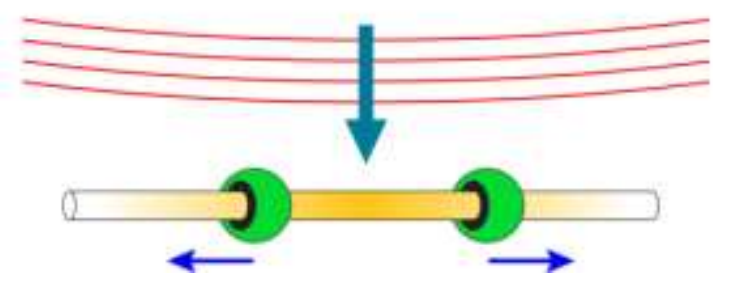

The setup includes beads on a stick, serving as a detector: The two beads can "[slide] freely (but with a small amount of friction) on a rigid rod. As the wave passes over the rod, atomic forces hold the length of the rod fixed, but the proper distance between the two beads oscillates. Thus, the beads rub against the rod, dissipating heat." 23

According to Feynman, the subsequent heating up indicates that the GW can do work: For conservation of energy to hold, whence should the gain in thermal energy stem, if not from the GW? We are thus licenced to ascribe GWs energy-momentum.

In order to discern more sharply a potentially problematic assumption underlying the Sticky Bead Argument, let us render it more quantitive with a simple damped spring. ${ }^{24}$ Consider two masses $m_{1}$ and $m_{2}$ placed on the $x$-axis and connected by spring of spring constant $k$. The spring is at equilibrium when the masses are separated by length $L$. Let $x$ measure the displacement of the masses w.r.t. this equilibrium. If a purely $\bigoplus$-polarised $\mathrm{GW}$ hits the system, the induced oscillations obey $\frac{d^{2} x}{d t^{2}}+2 \beta \frac{d x}{d t}+\omega_{0}^{2}=-\frac{1}{2} h_{\oplus} L \omega^{2} \cos \omega t$ with the characteristic frequency of the oscillator $\omega_{0}:=\sqrt{\frac{k}{\mu^{\prime}}}$ the reducedmass of the system $\mu:=\frac{m_{1} m_{2}}{m_{1}+m_{2}}$ and the damping parameter $\beta:=\frac{b}{2 \mu}$, where the dissipative force is assumed to be $F_{\text {diss }}=-b \frac{d x}{d t}$. The work done by the GW on the oscillator, averaged over a cycle of oscillation, can thus be determined to give $\left\langle W_{G W}\right\rangle=-\left\langle E_{\text {kin }}+E_{\text {pot }}\right\rangle=-\left\langle\frac{\mu}{2}\left(\frac{d x}{d t}\right)^{2}+\frac{k}{2} x^{2}\right\rangle=\beta \mu x_{\max }^{2} \omega^{2}$ with the resonant amplitude $x_{\max }=\frac{1}{2} \frac{h_{\oplus} L \omega^{2}}{\sqrt{\left(\omega_{0}^{2}-\omega\right)^{2}+4 \omega^{2} \beta^{2}}}$. This dissipated energy manifests itself as thermal energy.

The changes in total energy of the system, $E_{\text {kin }}+E_{\text {pot }}$, are counterbalanced by changes in the energy of the GW, which we can thus determine from the dissipation.

The Sticky Bead Argument appears compelling - once one accepts its two underlying premises:

transport would constitute a sufficient criterion for the reality of GWs, I do not regard it a necessary one: GWs in my opinion are real, and they manifest themselves as losses of matter energy-momentum - without, however, there being any energy-momentum exchange between the GW and matter; GWs exist, but need not carry energy-momentum.

${ }^{23}$ Feynman et al. 2002), p. xxv-xvi

${ }^{24}$ Cf. Anderson/Creighton (2012), pp. 65. (This is the idea underlying resonant mass or bar detector, e.g. Maggiore (2008), Ch. 8.) 
(1) General-relativistic correction terms on the internal structure of the stick (in particular its binding energy) are negligible. ${ }^{25}$ This is manifest in our qualitative version of the argument by the fact that the spring constant, which certainly depends on the internal structure of the spring, is assumed to be the same before and after the GW.

(2) Energy conservation holds: An increase of energy is always counterbalanced by a decrease of energy elsewhere. This principle was expressly invoked in both versions of the Sticky Bead Argument.

Neither premise, however, was affirmed without any further argument. Both may legitimately be challenged: Cooperstock and Tieu, for instance, have -pace Feynman ${ }^{26}$ - attacked (1) to the effect that they claim no heat transfer occurs, when properly modelling the sticky: "what has been overlooked is that the bar itself has been presumed to be unaffected by the gravity waves". ${ }^{27}$

Rather than dwelling on the details of a physically adequate modelling of the Sticky Bead Argument (and the sometimes underestimated subtleties of the interplay between gravity and electromagnetism ${ }^{28}$,) । want to focus on (2). Energy conservation may hardly be taken for granted. In fact, it is fair to say that the violation of energy-momentum conservation for nonflat spacetimes has long and widely been acknowledged in the GR literature. ${ }^{29}$

Therefore, even if (1) is warranted and we did register an increase in thermal energy of a Sticky Bead detector, we cannot infer a transfer of energy from the GW, so as to restore energy balance: Energy conservation simply does not hold in GR. Rather, the heating-up ex nihilo, the (quantifiable) violation of energy conservation upon a GW hitting a detector, would be merely another instance of the sundry conceptual revisions GR has enforced on physics.

To summarise: The cogency of the Sticky Bead Argument derives from premises that, albeit uncontroversial in Classical Mechanics, become highly controversial, if not downright false in GR.

Let us now move away from these more qualitative arguments to more systematic approaches to GW energy.

\section{II.3. Perturbative approach}

We encountered linearised GW theory in both the Argument from Kinetic Energy and the Sticky Bead Argument, where one expands the metric around a flat background. Perturbation

\footnotetext{
${ }^{25}$ Cf. Cooperstock/Tieu (2012), pp. 85

${ }^{26}$ Cf. Feynman, cited in Kennefick (2007), p. 136

27 Op.cit., p. 85

${ }^{28}$ Think, for instance, of the vexed question whether a point charge in free-fall radiates or not, e.g. Lyle (2008) By no means do I intend to disparage Cooperstock's efforts to rebut the Sticky Bead Argument. In fact, a satisfactory response to them is still pending, cf. Kennefick (2007), pp. 254.

${ }^{29}$ E.g. Eddington (1923), pp. 135 ; Schrödinger (1950), pp. 72, Weinberg (1972), p. 166, Misner et al. (1974), $\S 19.4$, Padmanabhan (2010), p. 213; Hoefer (2000), Lam (2011), Dürr (2017ab, ms)
} 
theory generalises this idea by decomposing the metric into a slowly-varying background and a fast-varying perturbation; the latter then is identified as the $\mathrm{GW}$ - a ripple of the spacetime. ${ }^{30}$ An object then emerges that appears to qualify naturally as representing the GW's energymomentum.

For simplicity, we restrict ourselves to vacuum solutions of the full Einstein Equations, $G_{a b}=$ 0 . Let there exist of suitable length (or time) scales of the variation. This enables us to decompose the metric into a background and small fluctuation components.

\section{Fig. 3:}

Characteristic length scales $\lambda$ of the GW and of the background curvature $L$, respectively

(picture taken from Straumann (2013), p. 246)

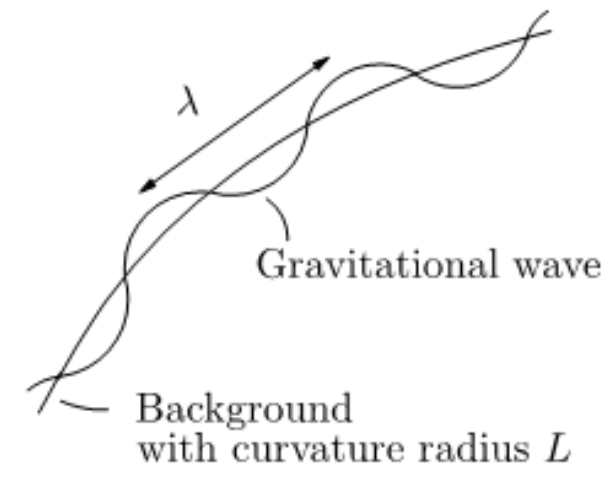

Applying the standard procedure for perturbation theory for the metric (with the formal bookkeeping parameter $\epsilon$ ),

$$
g_{a b}=g_{a b}^{(0)}+\epsilon g_{a b}^{(1)}+\epsilon^{2} g_{a b}^{(2)},
$$

the Einstein tensor can then be expanded up to $\mathcal{O}\left(\epsilon^{2}\right)$ as

$$
G_{a b}=G_{a b}^{(0)}\left[g_{a b}^{(0)}\right]+\epsilon G_{a b}^{(1)}\left[g_{a b}^{(0)}, g_{a b}^{(1)}\right]+\epsilon^{2}\left(G_{a b}^{(1)}\left[g_{a b}^{(0)}, g_{a b}^{(2)}\right]+G_{a b}^{(2)}\left[g_{a b}^{(0)}, g_{a b}^{(1)}\right]\right) \text {. }
$$

Here, the superscript " $(0)$ " denotes the unperturbed $\left(0^{\text {th }}\right.$ order) quantities and, correspondingly, "(1)“ the $1^{\text {st }}$ order perturbations, etc. The dependence of the $1^{\text {st }}$ order Einstein tensor, $G_{a b}^{(1)}\left[g_{a b}^{(0)}, g_{a b}^{(1)}\right]$, on the arguments in brackets denotes that it is built from $0^{\text {th }}$ and $1^{\text {st }}$ order terms of the metric.

In the expansion of the Einstein tensor, the first two terms describe the unperturbed background geometry, and the evolution of the perturbations on the background, i.e. the GWs, respectively. The last term is of principal interest for us: It describes how the $2^{\text {nd }}$ order perturbations are related to the background and $1^{\text {st }}$ order perturbations. Recast as

$$
G_{a b}^{(1)}\left[g_{a b}^{(0)} ; g_{a b}^{(1)}\right]=\frac{8 \pi G}{c^{4}} t_{a b}^{(\mathrm{eff})},
$$

with the "effective GW energy-momentum tensor" $t_{a b}^{(\mathrm{eff})}=-\frac{c^{4}}{8 \pi G} G_{a b}^{(2)}\left[g_{a b}^{(0)} ; g_{a b}^{(1)}\right]$ on the r.h.s., third term in the expansion of the Einstein tensor is susceptible to a natural elucidation: The $2^{\text {nd }}$ order perturbations of the metric are sourced by the effective GW energy-momentum, reflecting, as the standard explanation goes ${ }^{31}$, the back-reaction of the gravitational field upon

\footnotetext{
${ }^{30}$ Cf. Maggiore (2008), Ch. 1.4; Padmanabhan (2010), Ch. 9.5

${ }^{31}$ E.g. Hobson et al. (2008), Ch. 17.11
} 
itself; ${ }^{32}$ gravitational energy qua energy contributes to its own generation: "Gravity itself gravitates."

An immediate blemish tarnishes the effective GW energy-stress tensor $t_{a b}^{\text {(eff) }}$. For simplicity, let the spacetime background be flat. Then, the effective gravitational energy-stress pseudotensor is not invariant under the gauge transformations of the type $h_{\mu \nu} \rightarrow h_{\mu \nu}^{\prime}=h_{\mu \nu}$ $\partial_{(\mu} \xi_{v)}$. This lack of gauge-invariance can be cured by averaging over a 4-volume $\Delta \mathcal{V}$ of several wave lengths of the GW (but still smaller than the length scale of variation of the background): $: 33$

$$
T_{a b}^{(\mathrm{GW})}:=\left\langle t_{a b}^{(\mathrm{eff})}\right\rangle=\frac{1}{|\Delta \mathcal{V}|} \int_{\Delta \mathcal{V}} d^{4} x \sqrt{\left|g^{(0)}\right|} t_{a b}^{(\mathrm{eff})}
$$

(This ruse is seen as unproblematic, since perturbations can only be defined w.r.t. the typical length/time scales of the background.) This averaged GW energy-momentum then is gaugeinvariant: The changes of $t_{a b}^{\text {(eff) }}$ resulting from gauge transformations take the form of total divergences and are hence eliminated by the integration. The effective GW energymomentum $T_{a b}^{(\mathrm{GW})}$ has the following properties that at first blush commend it for an interpretation as encoding the energy-momentum of a GW:

(1) It transforms tensorially w.r.t. tensor transformations of the background metric $g_{a b}^{(0)}$. (Indices therefore are also raised and lowered w.r.t. to $g_{a b}^{(0)}$.)

(2) It is by construction symmetric (an important requirement for defining angular momentum).

(3) It obeys a generally covariant conservation law, $\nabla^{b}\left(T_{a b}+T_{a b}^{(\mathrm{GW})}\right)=0$ where the covariant derivative is defined w.r.t. the background metric $g_{a b}^{(0)}$.

(4) Like other energy-momentum tensors from classical field theories, it is quadratic in the dynamical components of the gravitational field, the GW field variables $g_{a b}^{(1)}$.

(5) It is regarded ${ }^{34}$ to originate in the non-linearity of the Einstein equations, supposed to reflect the fact that "gravity gravitates", with all forms of energy including gravitational energy itself act as a source for the gravitational field.

An equivalent re-formulation of the perturbative approach is possible in terms of a variational principle ("Isaacson's variational approach") ${ }^{35}$. The idea is to expand the action $S\left[g_{a b}+\right.$ $\left.h_{a b}\right]=\int d^{4} x \sqrt{\left|g\left[g_{a b}+h_{a b}\right]\right|} R\left[g_{a b}+h_{a b}\right]$ w.r.t. the perturbations $h_{a b}$ around the background $g_{a b}$, such that:

\footnotetext{
${ }^{32}$ For the sake of the argument, I grant that the gravitational field should indeed be identified with the metric eliding thereby the prolonged debate over this, cf. Read (2017, forth).

${ }^{33} \mathrm{Cf}$. Misner et al. (1973), §35.15

${ }^{34}$ Op. cit. pp. 486.

${ }^{35}$ Cf. Schutz/Ricci (2010), Sect. 4.2
} 


$$
\begin{aligned}
S\left[g_{a b}+h_{a b}\right]= & S\left[g_{a b}\right]+\int d x^{4} h_{a b} \frac{\delta(\sqrt{|g|} R)}{\delta g_{a b}} \\
& +\frac{1}{2} \int d x^{4}\left(\frac{\partial^{2}(\sqrt{|g|} R)}{\partial g_{a b} \partial g_{c d}} h_{a b} h_{c d}+2 \frac{\partial^{2}(\sqrt{|g|} R)}{\partial g_{a b} \partial\left(\partial_{e} g_{c d}\right)} h_{a b} \partial_{e} h_{c d}\right. \\
& \left.+\frac{\partial^{2}(\sqrt{|g|} R)}{\partial\left(\partial_{e} g_{c d}\right) \partial\left(\partial_{f} g_{c d}\right)} \partial_{e} h_{c d} \partial_{f} h_{c d}+2 \frac{\partial^{2}(\sqrt{|g|} R)}{\partial g_{a b} \partial\left(\partial_{e, f} g_{c d}\right)} h_{a b} \partial_{e, f} h_{c d}\right)+\mathcal{O}\left(h^{3}\right) .
\end{aligned}
$$

Let us denote the term in brackets in the last integral as the GW Lagrangian, as a function of the background metric and its perturbation, $32 \pi L_{(G W)}=32 \pi L_{(G W)}\left(g_{a b}, h_{a b}\right)$.

Given this GW Lagrangian, we can define an effective energy-stress tensor associated with the GW as a variational derivative w.r.t. the background metric:

$$
t_{(G W)}^{a b}=\frac{2}{\sqrt{|g|}} \frac{\delta}{\delta g_{a b}}\left(\sqrt{|g|} L_{(G W)}\right) .
$$

Averaging as before (in order to cure gauge-dependence), yields the same effective energymomentum:

$$
T_{(G W)}^{a b}=\left\langle t_{(G W)}^{a b}\right\rangle .
$$

The capacities of the perturbative approach remain limited, however, for reasons: The first one turns on its problematic reliance on conceptual prerequisites, not tenable at GR's fundamental level; the other one consists in the limited utility of perturbatively defined GW energy, tout court.

The perturbative approach explicitly presupposes a background-perturbation split, entering both the very construction of the energy-momentum from perturbative orders and the averaging procedure to overcome gauge-dependence.

Such a split is occasionally justified from a practical point of view, say for primordial GWs on an FLRW background, when one is dealing with distinguishable scales, $\lambda$ and $L$, over which the background geometry and the perturbation vary, $\lambda \ll L$.

A straightforward comparison of the orders of magnitude discloses, however, that "one cannot introduce the concept of a gravitational wave of arbitrarily large amplitude but varying at a length scale that is sufficiently small compared with the background scale of variation and develop a systematic perturbation theory". ${ }^{36}$ For, instance, at early times during cosmic inflation, the wavelength of GWs is smaller than the Hubble scale ("inside the horizon"); as inflation proceeds, the GW's wavelength redshifts and becomes eventually larger than the Hubble scale ("outside the horizon"). ${ }^{37}$ A GW thus cannot be fundamentally characterized as such a "ripple on a background". Such a split of the metric is artificial, however: There is only one metric, defying any intrinsically meaningful severing of "perturbations" from a "background".

\footnotetext{
${ }^{36}$ Padmanabhan (2010), p. 420; see also Padmanabhan (2004)

${ }^{37}$ Cf. Flanagan/Hughes (2005), Sect. 5.2
} 
I am not avowing that only fundamental quantities are conceptually kosher. ${ }^{38}$ My presently primary purpose is not to tackle when/how the introduction of higher-level concepts is justified and ontologically juicy; I merely point out what can be said by availing oneself only of the concepts fundamentally at our disposal in GR - and then show that the perturbative approach to $\mathrm{GW}$ energy treats these non-fundamental concepts in a way GR vetoes.

The best way thus to regard the perturbative approach is as describing the transition from a fundamental to a coarse-grained description ${ }^{39}$ - a tool convenient for approximations under certain conditions, but not a fundamental account. ${ }^{40}$

This might elicit the worry that such skepticism towards the background-perturbation split on the grounds of its non-fundamentality throws out the baby with the bathwater: Does such a "fundamentalism" not bereave us of the very concept of a GW altogether? Does the latter not presuppose a perturbative approach? One reply could be: There exist fundamental GWs, if by that one means exact wave-solutions of the Einstein Equations, such as the pp-wave metric. ${ }^{41}$ This may be seen as too restrictive, leaving almost no realistic cases of GWs. Hence, a second kind of reply is perhaps more satisfactory, disavowing that higher perturbative orders of the metric adequately characterise GWs: Rather, GWs should be identified with the Weyl tensor. ${ }^{42}$ I shall not dwell further on such semantic issues and, for the time being, merely assume that GWs are indeed only non-fundamental entities.

Should this non-fundamentality disconcert us? Albeit not in itself problematic, it is enmeshed in three problematic facets of the perturbative approach.

The first one is related to the way the perturbative approach breaks GR's general covariance: The effective energy-momentum transforms tensorially only under tensor transformations of the background metric, not under general transformations. GR's conceptual framework, however, gainsays such a preferred status of any background.

A second problem arises, when $T_{a b}^{(\mathrm{eff})}$ (or $t_{a b}^{(\mathrm{eff})}$ ) is introduced to explain how the $2^{\text {nd }}$ order perturbation propagates on the background. This suggests that whereas the Einstein Equations suffice to explain the behaviour of the background metric, the behaviour of the perturbations calls for an explanation transcending the Einstein Equations. One thus imputes the perturbations a different status than the background - an explanatory asymmetry that the fundamental level, on which no perturbative order of the metric is privileged, disclaims. For a flat background this asymmetry is even more striking: Then the fact that the propagation of the second order-perturbations is seen in explanatory need amounts to postulating that the non-linear terms of GR call for an explanation of a type that the linear ones do not. But this

\footnotetext{
${ }^{38}$ E.g. Wallace (2012), Ch. 2, who pleas for taking also non-fundamental objects ontologically seriously.

${ }^{39}$ Cf. Maggiore (2008), Ch. 1.4.2

${ }^{40}$ The averaging over several wavelengths we prescribed above in order to overcome the gauge-dependence of the effective GW energy-momentum is in fact a special case of renormalization group transformations, familiar from effective field theories to describe transitions from different levels of description, cf. Peskin/Schroeder (1995), Ch. 12.

${ }^{41}$ Cf. Misner et al. (1973), Ch.35.9, 35.10; 35.11.

${ }^{42}$ E.g. Padmanabhan (2010) Ch. 5.5.3; 6.3
} 
simply ignores the fact the GR is fundamentally non-linear ${ }^{43}$ - one just cannot privilege the linear parts of an essentially nonlinear theory. ${ }^{44}$

A third problem in the way the perturbative approach utilises the background-perturbation split becomes evident in Isaacon's variational approach: In order to obtain the correct field equations, both the background metric and the perturbation must be treated as independent variables, so that the variation w.r.t. each vanishes. Thus, the perturbation is effectively treated like a matter field (as opposed to the background metric, which presumably retains its orthodox spacetime interpretation), with its own energy-momentum tensor. ${ }^{45}$ Such a reclassification of a perturbative order of the spacetime metric to an independent matter field certainly is a substantive deviation from GR's conceptual basis.

After such conceptual worries, let us finally turn to two practical shortcomings that curb the value of any applications of GW energy, based on the perturbative approach: ${ }^{46}$

To begin with, the perturbative approach does not provide any notion of a "gravitational energy of the system". (The quotation marks are to signify that it is not obvious that it even makes sense in $\mathrm{GR} .{ }^{47}$ ) Consequently, the perturbative approach falls short of paradigmatic applications, such as binary systems, "gravitational energy" of which is depleted by the GWs. Furthermore, a perturbatively defined GW energy is also too crude to deliver the flux of angular momentum, important for the correct description of millisecond pulsars, whose rotation rate increases due to a transfer of angular momentum from the accretion disk surrounding the pulsar. ${ }^{48}$ Consequently, since the primary raison d'être of a definition of GW energy consists in its astrophysical utility, the perturbative road seems to be a blind alley from the start.

Before reconnoitring the most systematic and hence promising path to GW energy, let us summarise the preceding critique of the perturbative approach: It presupposed a fundamentally ungrounded background-perturbation split; based on this, GW energy was introduced and used in a way at variance with GR's conceptual framework. Last, not least we also found the perturbative approach as a whole unsuitable for astrophysical applications, which renders the value of a perturbatively defined GW energy-momentum questionable altogether.

\section{II.4. The Noetherian perspective}

\footnotetext{
${ }^{43}$ Cf. Aldrovandi et al. (2007)

${ }^{44}$ Hence, one should generally restrain one's penchant to compare GR with the linear Maxwell theory; any analogies should preferably be drawn with the likewise genuinely non-linear vectorial Yang-Mills-type theories, cf. Deser (1970). It is noteworthy that in such Yang-Mills theories energy is localisable: Thus, the problems with localising gravitational energy in GR does not originate in its nonlinearity per se, as sometimes claimed.

${ }^{45}$ Schutz/Ricci (2010), p. 41 are explicit about this.

${ }^{46}$ Cf. Poisson/Will (2014), Ch. 12.2.5

${ }^{47}$ E.g. Hoefer (2000), Dürr (2017)

${ }^{48}$ Cf. Poisson/Will (2014), Ch. 12.2.4
} 
In this subsection, I shall examine the presumably most systematic approach to GW energymomentum, via (a suitable generalisation of) Noether's Theorems, which treats GR's metric completely analogously to other classical fields.

One of GR's characteristics from the gauge theoretical point of view, to the fecundity of which I already alluded, is its general covariance (GC), the invariance under general diffeomorphisms. ${ }^{49} \mathrm{GC}$ as a local symmetry of the Einstein-Hilbert action $\int d^{4} x \sqrt{|g|} R$ lends itself to an application of Noether's $2^{\text {nd }}$ Theorem, linking local gauge symmetries and conserved quantities. ${ }^{50}$

For GR, a generalisation of Noether's Theorem is more apposite: Firstly, the results of the Noether Theorem are gauge-dependent. In light of the havoc laxness regarding gaugedependence has wreaked in the history of $\mathrm{GWs}^{51}$, we should be particularly circumspect in assuring ourselves of the gauge-invariance of our results. A second (and related) shortcoming of Noether's Theorem consists in that it only exploits the information encoded in the vanishing of the interior contributions to the Variational Problem. This seems unduly restrictive: Why assume a priori that GR's metric suitably peters out at infinity so that contributions to the boundary can be discarded without impunity? (One can easily construct even exact GW solutions as counterexamples.) The Klein-Utiyama Boundary Theorem addresses both issues. ${ }^{52}$ It purports the following:

Consider the action $S\left[\psi_{i}\right]=\int d^{4} x \mathfrak{L}\left(\psi_{i}, \partial \psi_{i}, x\right)$ of the generic fields $\psi_{i}$ be invariant (up to a surface term) under an infinite-dimensional Lie group $G_{\infty, \rho}$ of transformations that smoothly depend on $\varrho$ functions $p_{\alpha}\left(x^{a}\right)$ and their derivatives ${ }^{53} \partial_{\beta} p_{\alpha}\left(x^{a}\right)$ and give rise to the variation of the dynamical fields $\psi_{i}$ (of generic tensorial type), $\delta \psi_{i}=\sum_{\alpha}\left(a_{\alpha i} \Delta p_{\alpha}+b_{\alpha i}^{c} \partial_{c} \Delta p_{\alpha}\right)$, where $a_{\alpha i}$ and $b_{\alpha i}^{\mu}$ are coefficient functions that depend on $x^{a}, \psi_{i}$ and $\partial_{c} \psi_{i}$. (The $\Delta p_{\alpha}{ }^{\prime}$ s indicate that we are taking infinitesimal $\mathrm{p}_{\alpha}{ }^{\prime} \mathrm{s}$.)

Then there exist three sets of $\varrho$ relationships:

- $\sum_{i} a_{\alpha i} \frac{\delta \mathfrak{L}}{\delta \psi_{i}} \equiv-\sum_{i} \partial_{c}\left(a_{\alpha i} \frac{\partial \mathfrak{L}}{\partial\left(\partial_{c} \psi_{i}\right)}\right)$

- $\sum_{i} b_{\alpha i}^{\mu} \frac{\delta \mathfrak{R}}{\delta \psi_{i}} \equiv-\sum_{i}\left(a_{\alpha i} \frac{\partial \mathfrak{L}}{\partial\left(\partial_{\mu} \psi_{i}\right)}+\partial_{v}\left(b_{\alpha i}^{\mu} \frac{\partial \mathfrak{R}}{\partial\left(\partial_{\nu} \psi_{i}\right)}\right)\right)$

- $\sum_{i}\left(b_{\alpha i}^{v} \frac{\partial \mathfrak{R}}{\partial\left(\partial_{\mu} \psi_{i}\right)}+b_{\alpha i}^{\mu} \frac{\partial \mathfrak{L}}{\partial\left(\partial_{\nu} \psi_{i}\right)}\right) \equiv 0$

Here $\frac{\delta \mathfrak{Q}}{\delta \psi_{i}}$ denotes the variational derivatives, i.e. the familiar Euler-Lagrange expressions. Germane to our purposes is the first identity: After some rearranging, one can infer from it that the Noetherian 4-current

\footnotetext{
${ }^{49}$ As a symmetry group, the role of, say, the $U(1)$ gauge group in electrodynamics, in GR is taken over by the (non-Abelian) diff( $\mathcal{M})$ group, e.g. Straumann (2013), Ch. 3.3; 3.6.

${ }^{50}$ E.g. Maggiore (2008), Ch. 2; Brading/Brown (2000); Brown/Brading (2002)

${ }^{51}$ Cf. Kennefick (2007), esp. Ch. 4,5

${ }^{52}$ Cf. Brading/Brown (2000); Ohanian (2013), Appendix 1 recapitulates all the steps.

${ }^{53}$ For simplicity, a restriction is made to first derivatives.
} 


$$
j_{k}^{\mu}:=-\sum_{i}\left\{\frac{\partial \mathfrak{L}}{\partial\left(\partial_{\mu} \psi_{i}\right)} \frac{\partial\left(\delta_{0} \psi_{i}\right)}{\partial\left(\Delta p_{k}\right)}++\mathfrak{L} \frac{\partial\left(\delta x^{\mu}\right)}{\partial\left(\Delta p_{k}\right)}-\frac{\partial\left(\Delta \Lambda^{\mu}\right)}{\partial\left(\Delta p_{k}\right)}\right\}
$$

(with the terms $\Lambda^{\mu}$, arising, when the action is not strictly invariant, such as in the case of GR) can be brought into the following form:

$$
j_{k}^{\mu}=b_{k i}^{\mu}\left(\frac{\partial \mathfrak{Q}}{\partial \psi_{i}}-\partial_{v} \frac{\partial \mathfrak{Q}}{\partial\left(\partial_{\mu} \psi_{i}\right)}\right)+\partial_{\nu} U_{k}^{[\mu \nu]}
$$

Here, the last term, $U_{k}^{[\mu \nu]}$, is a so-called superpotential, antisymmetric in its upper indices. ${ }^{54}$ Let us now apply Klein-Utiyama Theorem to GR with the (dynamically equivalent ${ }^{55}$ ) truncated Einstein-Hilbert (" $Г \Gamma^{\prime}$ ) Lagrangian

$$
\overline{\mathcal{L}}=2 g^{a b} \Gamma_{a[b}^{d} \Gamma_{c] d}^{c} .
$$

The last two identities of the Klein-Utiyama Theorem yield some interesting implications for GR's matter field equations. ${ }^{56}$ More important for our present purposes is the equation the first identity of the Klein-Utiyama Theorem, together with the Einstein Equation, entails:

$$
\sqrt{|g|}\left(T_{a}^{b}+t_{a}^{b}\right)=\partial_{c} \mathfrak{B}_{a}^{[b c]} .
$$

Here $t_{a}^{b}:=\frac{1}{\sqrt{|g|}}\left(-\overline{\mathcal{L}} \delta_{a}^{b}+\frac{\partial \overline{\mathcal{L}}}{\partial\left(\partial_{b} g_{d e}\right)} \partial_{a} g_{d e}\right)$ denotes the so-called Einstein pseudotensor ${ }^{57}$ and $\mathfrak{W}_{a}^{[b c]}$, again antisymmetric in its upper indices.

The Einstein pseudotensor corresponds to canonical gravitational energy-momentum, as we would expect it from other field theories. This suggests that $\mathfrak{T}_{a}^{b}:=T_{a}^{b}+t_{a}^{b}$ should be interpreted as the total (matter cum gravitational) energy-momentum.

Thanks to the superpotential's antisymmetry in its upper indices, total energy-momentum obeys a continuity equation:

$$
\partial_{b}\left(\sqrt{|g|} \mathfrak{T}_{a}^{b}\right)=\partial_{b, c} \mathfrak{M}_{a}^{[b c]} \equiv 0 .
$$

Albeit not a tensor equation, the continuity equation holds in every coordinate system and is intuitively construed as a conservation principle, reflecting the absence of sinks and sources of the total energy-momentum.

For the moment, let us assume that the Klein-Utiyama Theorem is applicable without any further technical problems (related to, say, the convergence of the integrals). Then, what sense are we to make of the result it yields, i.e. its canonical gravitational energy-momentum pseudotensor? In particular: To what extent are we licenced to interpret it realistically, i.e. ascribe it a status other than merely formal?

\footnotetext{
${ }^{54}$ The details of superpotentials need not detain us here, cf. Trautmann (1962).

${ }^{55}$ E.g., Hobson et al (2008), Ch. 19.19

${ }^{56}$ Brown/Brading (2006), sect. IV

${ }^{57}$ For a handier form, cf. Dirac (1975), Ch. 32
} 
The question comprises two facets: The first one concerns problems of the interpretation of the Noetherian energy-momentum 4-currents ("local energy-momentum"); the second one questions whether these 4-currents give rise to global (integral) quantities, identifiable as gravitational energy-momentum.

Regarding the status of the gravitational energy-momentum 4-current, a straightforward realist interpretation is impeded by four difficulties:

- Gauge-dependence: Being a pseudotensor, the energy-momentum 4-current does not transform tensorially under arbitrary coordinate transformations; its invariance is only preserved under linear transformations. However, in spacetimes other than Minkowski space, linear transformations are no longer privileged. The object denoted by the pseudotensor thus depends on the arbitrary, conventional choice of a coordinate system - in contrast to the invariance one would naturally demand of a physical quantity. ${ }^{58}$

Weyl neatly summarises the pickle: "Indeed all the [pseudotensor components] can, through a suitable choice of a coordinate system, be made to vanish; [...] on the other hand one obtains [pseudotensor components] that are different from zero in a 'Euclidean', completely gravity-free world by using a curvilinear coordinate system, where it seems pointless to speak of gravitational energy." 59

- Index-nonsymmetry: The pseudotensor is not symmetric in its indices, marring its physical suitability for defining angular momentum.

One can cure this blemish by the standard Belinfante- Rosenfeld technique. But this technique requires privileging a symmetry in a manner prima facie not justified within GR. ${ }^{60}$

- Ambiguity: The canonical energy-momentum 4-current above is defined only up to a choice of a superpotential. But different choices of superpotentials - none in itself less apt than any other - yield different pseudotensors, potentially associated with different global/integral energy-momentum distributions. The pseudotensor thus is vastly underdetermined. ${ }^{61}$

- Physical significance: Klein adverted to the fact that the formal continuity equation, deduced from the Klein-Utiyama Theorem, holds without any need to take a divergence: ${ }^{62}$ Given the Einstein Equations, its status thus rather resemblance that of a mathematical identity. Does this mean, as Klein believed, that the continuity equation is vacuous in content and cannot be conferred upon a realist meaning?

In light of such qualms, ought we perhaps to follow Weyl's suggestion that a realist interpretation of the Klein-Utiyama-Noetherian results be reserved for the global quantities, associated with the 4 -currents? ${ }^{63}$

\footnotetext{
${ }^{58}$ E.g. Vollmer (2010)

${ }^{59}$ Weyl (1923), p. 273

${ }^{60}$ Cf. Leclerc (2006)

${ }^{61}$ In fact, (uncountably) infinitely many choices are possible, cf. Bergmann (1958), Komar (1959).

${ }^{62}$ Cf. Brading (2005)

63 "Still, from a physical point of view, it seems meaningless to introduce the [pseudotensor] as energy components of the gravitational field, for they form neither a tensor nor are they symmetric [...]. Even if the
} 
Again, the devil lurks in the detail. For the integrals to be well-defined, the metric must satisfy certain coordinate conditions ("asymptotic flatness"). This raises a number of delicate points: Do the required coordinate conditions amount to an unnatural gauge fixing, i.e. do they artificially pick out certain coordinate systems? How to justify asymptotic flatness? How physically plausible is it? How restrictive are the constraints it imposes on the space of admissible spacetimes? (The to-date most successful cosmological $\Lambda \mathrm{CDM}$ model of the universe, for instance, fails to be asymptotically flat.) How much do the quantities, thus formally defined, meet our desiderata for gravitational energy-momentum in a substantive sense (e.g. via a sufficient family resemblance with notions of field energy in other field theories)? What is the relation to other alternative proposals for capturing general-relativistic gravitational energy-momentum?

None of these issues pertains to GW energy-momentum specifically; rather, they bedevil gravitational energy-momentum in GR more generally. It is beyond the present paper's ambit to enter this thorny debate. Yet, the lesson seems clear: We must countenance the controversial nature of gravitational (and, a fortiori, GW) energy-momentum obtained via the Klein-Utiyama theorem; the Noetherian approach to GW energy-momentum ultimately prompts more questions than it conclusively answers.

\section{Are binary systems evidence for GW energy?}

In this section, I shall analyse the received explanation of binary systems, and whether it is sound. I contest this and proffer an alternative and in several regards superior explanation exclusively in terms of the general-relativistic Equations of Motion and the Einstein Equations.

\section{III.1. The standard view}

The dynamical standard interpretation of the binary problem explains the orbital decay via the system's total energy being carried away by the emitted GWs.

More precisely ${ }^{64}$, the standard interpretation starts from an energy balance of the (symbolic) form $\dot{E}=-L$, with the change in a system's total energy on the I.h.s. being compensated by the energy-flux on the r.h.s., the energy-momentum radiated away.

Such an energy balance is provided by a realist interpretation of the continuity equation for total energy-momentum, based on the energy-momentum tensor of matter and

differential relations [i.e. the pseudotensor-based continuity equation, P.D.] are without any real physical meaning, they do give rise to an invariant statement of conservation via integrating over an isolated system." Weyl (1923), p. 273

${ }^{64}$ Cf. Poisson/Will (2014), Ch. 6-12 
pseudotensor encoding gravitational and GW energy-momentum. A standard choice is the index-symmetric pseudotensor proposed by Landau and Lifshitz: ${ }^{65}$

$$
\partial_{b}\left(|g|\left(T^{a b}+t_{(L L)}^{a b}\right)\right)=0 .
$$

Integration over a 3-region $\mathcal{V}_{3}$ then yields the corresponding total energy-momentum $P^{a}=$ $\left(E / c, P^{i}\right)$ :

$$
P^{a}=\frac{1}{c} \int_{\mathcal{V}_{3}} d^{3} x|g|\left(T^{a 0}+t_{(L L)}^{a 0}\right)
$$

(Recall that this total energy-momentum transforms like a Minkowski tensor at infinity.) It is sometimes more convenient to express the volume integrals as surface integrals, exploiting that the continuity equation can be re-written in terms of Landau and Lifshitz's superpotential $H^{\alpha \mu \beta \nu}$ as

$$
P^{a}=\frac{c^{3}}{16 \pi G} \oint_{\partial \mathcal{V}_{3}} d \sigma_{k} \partial_{\mu} H^{\alpha \mu 0 k} .
$$

The next step consists in approximately evaluating this balance equation for a suitable model of the binary system for the regime of interest, most commonly the far fields of a compact source (whose energy content is dominated by its rest mass). The standard framework for such an evaluation is the so-called PPN formalism, which essentially expands the metric in orders of the small ratio between the bodies' velocity and the speed of light.

Expanding thus the balance equation to leading (viz. $5^{\text {th }}$ ) order of the onset of GW effects, we extract those parts of the energy-momentum that are identified with the radiative degrees of freedom. We then lump them into what we shall refer to as the "GW luminosity", i.e. the energy-momentum flux due to GW emission. This GW luminosity forms the r.h.s. of the energy balance, $\dot{E}=-L$, the dissipated energy.

The residual terms in the expression for the total energy-momentum are then identified as the "total energy of the system", reproducing in leading order the total (kinetic plus gravitational) energy of the Newtonian 2-body/Kepler problem. For a higher accuracy, nextleading order terms can also be consistently incorporated, yielding correction terms to the Kepler potential (responsible for, say, the perihelion shift), as well as only velocity-dependent terms, perhaps best construed as corrections to kinetic energy.

Solving the Equations of Motion, one then finds that the physical distance between the binaries is decreasing: Like radiative losses in electromagnetic theory (or a mechanical system with friction), the emission of GWs dissipates energy from the system, causing its orbital decay: The system's motion is no longer bound; the energy loss destabilizes it.

For the sake of concreteness ${ }^{66}$, consider a system of two point-particles of equal mass $M$, rotating around their centre point with constant angular velocity $\omega$ and with the coordinate

\footnotetext{
${ }^{65}$ E.g. Landau/Lifshitz (1971), §101

${ }^{66}$ E.g. Hobson et al. (2006), Ch. 18
} 
distance $a$ between them (imagine them to be connected by a light rod). The corresponding balance equation in leading order reads thus:

$$
\frac{1}{2}(2 M) v^{2}-\frac{G M}{2 a}=-\frac{128 G}{5 c^{5}} M^{2} a^{4} \omega^{6} .
$$

The I.h.s. is corresponds to the system's total energy; the r.h.s. describes the energy drain via emission of GWs. From this balance equation, one can derive an equation for the spin-up, i.e. the rate of change of the orbital period $P$, directly accessible to observation:

$$
\dot{P}=-\frac{96}{5} 4^{\frac{1}{3}} \pi\left(\frac{2 \pi G M}{P}\right)^{5 / 3} \text {. }
$$

The logical structure of the standard explanation of the binary problem can thus be explicated via the following three statements:

(1) Along the bound or scattered orbits of a 2-body system, i.e. if the orbit prescribed by the Equations of Motion does not decay, the system's total (i.e. gravitational cum matter) energy-momentum is conserved.

(2) The binary system's total energy-momentum is conserved.

(3) GWs carry away energy-momentum from the system.

Given (1), it follows from (2) and (3) that orbits decay: Because GWs carry energy away from the system, the otherwise bound orbits decay. GW energy in (3), together with the principle (1), here serves as the explanans for the decrease in total energy, which in turn via modus tollens of (1) entails the explanandum orbital decay ( $\mathcal{E}$ "):

$$
(3) \rightarrow \neg(2) \stackrel{(1)}{\rightarrow} \mathcal{E} \text {. }
$$

\section{III.2. Criticism}

Let us now critically examine this standard explanation. I submit, it is fraught with three types of defects: Firstly, it contains assumptions and concepts that are at odds with GR; secondly, the third premise is unnecessarily strong, and thirdly, the crucial premise (1) remains unjustified.

The first strand of criticism applies to all three steps: (1), (2) and (3) all involve the concepts of GW energy or gravitational energy. Both are highly controversial - in fact, we saw in sect. II that the standard arguments cited for the energy ascribable to GWs are spurious - and have even been argued to be alien to GR's conceptual framework: ${ }^{67}$ After all, GR is a theory that "geometrises away" gravity, i.e. re-conceptualises gravity as a manifestation of a nonMinkowskian/Newtonian inertial structure. ${ }^{68}$ If thus GR "geometrises away" the gravitational

\footnotetext{
${ }^{67}$ E.g. Hoefer (2000), Curiel (2011), Petkov (2017)

${ }^{68}$ An elegant derivation of the Einstein Equations that makes this geometrisation explicit is found in Geroch (2013), Ch. 19
} 
force, one should expect this to compromise the idea of gravitational energy-momentum, as well. ${ }^{69}$

Furthermore, the standard explanation tacitly assumes that one can clearly sever the system's gravitational energy-momentum from the GWs'. While this is true for the lowest perturbative orders, in higher orders the radiative and the static gravitational degrees of freedom are inextricably intertwined. In consequence, the Equations of Motion defy a standard Lagrangian or Hamiltonian formulation, undermining the notion of the system's total energy-momentum.

If one wants to introduce energy for (gravitationally or electromagnetically) radiating systems, one needs to resort to a principle of energy-momentum conservation: The radiated energy is constructed such that energy conservation is restored. But in GR this principle becomes doubtful: In fact, many have argued that in GR matter energy-momentum just fails to be generically conserved. Energy conservation, however, lies at the heart of a realist interpretation of the continuity equation $\partial_{\mu}\left(|g|\left(T^{\mu \nu}+t_{(L L)}^{\mu \nu}\right)\right)=0$. But such a straightforward realist interpretation, which takes the pseudotensor to represent gravitational or GW energy, is obstructed by in particular the pseudotensor's gaugedependence.

A second line of attack aims at assumption (3) as unnecessarily strong: It is not necessary to demand that energy be carried away. For the argument to go through, it suffices to accept a decrease in the system's total energy-momentum - without this loss in energy-momentum being compensated for by equally real energy ascribable to the GW and propagating unintermittently from one place to another. What matters is only the violation of energy conservation; the transport of the "missing" energy is irrelevant.

One may well ask: What is the difference between violation of energy conservation and the "missing energy" being carried elsewhere? After all, as Curiel has pithily remarked: "One cannot tag hunks of energy as one can hunks of cheese, and so one cannot identify the energy that this system lost with the energy that that one gained in the same way one could if one were talking about cheese." 70 The choice between failure of energy conservation and its transference elsewhere hinges on the status of the conservation law to which the second option appeals in order to justify the postulated energy transport.

Three things can be said in this regard: Firstly, as I mentioned already, within GR it is widely acknowledged that the failure of the vanishing covariant divergence of the energy-momentum tensor, $\nabla_{a} T^{a b}=0$, to yield a conserved global/integral quantity simply reflects that energy conservation no longer holds ${ }^{71}$ - a line less revisionary than may appear at first blush: After all, the special-relativistic conservation laws for energy-momentum and angular momentum depend on the 10 Killing vectors of Minkowski space. Generic GR spacetimes, by contrast, lack any symmetries. So, absent such symmetries, why should we expect energy-momentum

\footnotetext{
${ }^{69}$ E.g. Norton (2012), pp. 19

The argument carries over to Newton-Cartan Theory, the geometrized version of Newtonian Gravity, cf. Dewar/Weatherall (2017).

${ }^{70}$ Curiel (2011),

${ }^{71}$ E.g. Carroll (2010)
} 
conservation to hold? Secondly, there does not exist any conceptually unproblematic way to express the dissipated energy: The most prominent way, for instance, via pseudotensors, suffers from numerous problems that obviate any straightforward realist interpretation. By contrast, the energy emitted by an electromagnetically radiating system allows for an innocuous tensorial representation. Indeed, there is a large consensus regarding the "nonlocalisability" of the dissipated energy: It does not seem to be possible to specify where in spacetime this GW energy resides. ${ }^{72} \mathrm{~A}$ third and last aspect germane to adjudicating between energy non-conservation and energy transport via GWs is the explanatory surplus value of the latter choice: Does postulating GW energy transport help us explain anything better? Should this be the case, an inference to the best explanation may abet that energy-momentum nonconservation should best be accounted for in terms of GW energy-momentum transport.

It is to this question I shall now turn: Is it possible to devise a perhaps better explanation of the orbital decay?

\section{III.3. A dynamical explanation}

To this end, let us revisit premise (1), pivotal to the standard explanation. How to vindicate it? Why believe that if the particles follow the scattered or bound orbits, prescribed by the Equations of Motion, the system's total energy is conserved?

The answer is: Because the theory's dynamics, i.e. the Equations of Motion (EoMs) - I use the term synonymously with "field equations"-, tell us so: In Newtonian Gravity, energy is generally conserved and the EoMs imply that orbits of celestial bodies are either hyperbolic ("scattered") or elliptical ("bound"): An object cannot spiral into the centre without energy dissipation, e.g. via tidal friction.

Why should we assume (1) also holds in GR? I think we just should not: The EoMs simply dictate that the binary pulsars' orbit decay. There is no need to invoke any quantity to explain the deviation from bound orbits. Why assume that such a deviation from bound orbits mandates an explanation? If one were to maintain (1), one would pick out from the full Equations of Motions those parts that describe conservative systems, i.e. systems whose energy is conserved; the deviation from the orbits of these conserved systems would then be explained in terms of the energy losses via GWs. One thus splits the EoMs into a conservative and a non-conservative part ("cEoMs") and treats the former as explanatorily distinguished: The deviation of the system's orbits from the ones obtained from the cEoMs calls for an explanation in terms of energy losses, whereas the conservative orbits are seen as the explanatory default motion. But what vindicates such a split? The reason seems to be simply habituation from pre-GR physics (where energy-momentum conservation is, of course, valid and ubiquitously useful): That all systems are conservative, unless some friction or radiation dissipates energy, has been so deeply engrained in our physical intuition that it has ossified

\footnotetext{
${ }^{72}$ E.g. Misner et al. (1973), p. 467
} 
into dogma. But if its plausibility presupposes the validity of energy-momentum conservation, and the latter needs to be jettisoned in GR, the justification of (1) lapses.

Rather, GR compels us just to accept that no bound solutions of the general-relativistic 2-body problem exist. ${ }^{73}$ As Cooperstock and Tieu put it, "on this basis, the period-changing binary pulsar is simply manifesting its conformity with the mathematical demands of Einstein's General Relativity rather than the preconceptions regarding energy." 74

It thus seems apposite to supplant (1) by the following principle, a direct consequence of the EoMs and the Einstein Equations:

$\left(1_{G R}\right)$ If a two-body system is emitting GWs, its orbits are in-spiralling.

By the same token, let us supplant (2) by simply accepting GR's failure of energy-momentum conservation. In order to eschew the problems associated with gravitational energy, the reference to total energy-momentum must be dropped:

$\left(2_{G R}\right)$ In generic (non-flat) spacetimes, matter energy-momentum is not conserved.

Since (3) turned out to be unnecessarily strong, we replace its reference to the purported energy-momentum transport by merely acknowledging that in spacetimes with GWs systems lose energy:

(3GR) GWs deplete a system's (matter) energy-momentum.

This principle $\left(3_{G R}\right)$ is obviously a special case of $\left(2_{G R}\right)$, since a spacetime with a $G W$ is by definition non-flat and hence displays energy-momentum non-conservation.

While both $\left(2_{G R}\right)$ and $\left(3_{G R}\right)$ are true, we do not need them for the explanation of the orbital decay (as before: " $\mathcal{E}$ ") as independent principles: They follow from the EoMs. (1 $1_{\mathrm{GR}}$ ) on its own affords us with an explanation that draws only on bona fide GR concepts and assumptions: Because the system emits GWs, its orbits are inspiralling.

The explanans now is the GW emission simpliciter, itself rooted in the specificities of the energy-momentum distribution. Since $\left(1_{G R}\right)$ is a consequence of the EoMs, our explanation is a special case of an explanation employing the EoMs: We can thus hone it by replacing $\left(1_{G R}\right)$ by the EoMs and specifying the antecedent matter conditions (QUA) that generate GWs, viz. the time-variation of its matter quadrupole moment tensor $I^{i j}=\int d^{3} y T^{00}(c t, \vec{y}) y^{i} y^{j}$.

The explanation we have thus arrived at makes only reference to a condition on the matter energy-momentum (QUA), the Einstein Equations (EEs) and the EoMs; instead of GW energy and (1) in the standard explanation, the explanantia are the EoMs and the matter energymomentum distribution as the antecedent conditions: Because the quadrupole moment of the matter energy-momentum distribution varies in time (QUA), the EoMS imply that the system's orbits decay (as before, denoted by " $\mathcal{E}^{\prime \prime}$ ):

\footnotetext{
${ }^{73}$ Cf. Papapetrou $(1957,58)$

${ }^{74}$ Cooperstock/Tieu (2012), p. 83
} 


$$
(\mathrm{QUA}) \stackrel{(E o M s) \&(E E s)}{\longrightarrow} \mathcal{E} .
$$

I shall call this the "dynamical" explanation of the orbital decay, as it employs only the dynamics encoded in the EoMs.

Note that in the dynamical explanation, GWs no longer play any explanatory role: The emission of GWs and the orbital decay share a common cause in the antecedent matter condition:

$$
(\mathrm{QUA}) \stackrel{(E o M) \&(E E s)}{\longrightarrow} \mathcal{E} \& \mathrm{GW} \text { emission. }
$$

By means of illustrating the dynamical interpretation, it is illuminating to address now Petkov's recent objection ${ }^{75}$ to the standard interpretation of binary systems on the grounds that "[...] [it] contradicts general relativity, particularly the geodesic hypothesis [...], because by the geodesic hypothesis the neutron stars, whose worldliness had been regarded as exact geodesics [...] move by inertia without losing energy since the very essence of inertial motion is motion without any loss of energy." 76

Glossing over mathematical qualms about the use of point-particles in $\mathrm{GR}^{77}$, the dynamical interpretation resolves the conflict Petkov perceives: W.r.t. the metric the EEs of the orbiting pulsars dictate, the pulsars indeed follow geodesics which, in contrast to the rectilinear ones in Minkowski spacetime, are spiraling-in towards each other: The 4-dimensional view of the binaries is that of a double helix whose radius decreases with time. This helical shape of the binaries' worldlines is a brute fact of GR's spacetime geometry (more precisely: GR's inertial structure, encoded in the affine connection compatible with the metric): The orbits of the 2body problem, i.e. the three-dimensional projection of the two world lines onto planes of simultaneity, just fail to be stable. The dynamical explanation expressly boycotts the identification of the system's total energy-momentum with the energy-momentum constructible from the conservative parts of the dynamics. ${ }^{78}$ In fact, the dynamical explanation does not even need any notion of energy-momentum other than the one of matter (which turns out to be not conserved): For the point-particles in free-fall this energy coincides with their rest mass - and is indeed conserved. In summary, Petkov's diagnosis of inconsistency of the standard explanation rests on the premise that the orbits of the general-relativistic 2-body problem must be bound and that the inspiral must be explained in terms of energymomentum transport. The dynamical explanation just denies these assumptions as unwarranted by a proper conceptual analysis of GR.

\footnotetext{
${ }^{75}$ In fact, Bondi had voice the same argument earlier, cf. Kennefick (2007), p. 200

${ }^{76}$ Petkov (2017), p. 11 (Petkov's emphasis)

${ }^{77}$ Cf. Straumann (2013), Ch. 6.4,6.5

${ }^{78}$ More precisely, on the standard explanation, one would define the system's energy the energy associated with the Einstein-Infeld-Hoffmann Lagrangian, e.g. Straumann (2013), Ch. 6.5
} 
Fig. 3:

Helical worldline of one of the binary partners. One spatial dimension is suppressed.

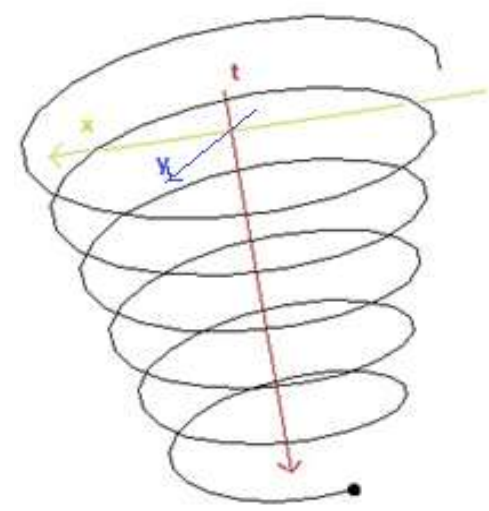

\section{III.4. Dynamical vs. standard interpretation}

We are now in a position to compare the standard and the dynamical interpretation to each other: How does the dynamical explanation fare vis-à-vis the one involving GWs dissipating gravitational (binding) energy from the system? I submit, the dynamical one is superior on grounds of parsimony, universal scope and unificatory power; the merits of the standard explanation, by comparison, are limited to its familiarity to pre-GR concepts and principles.

For reasons of space, my discussion is confined to the binary system's problem of motion, thereby setting aside the question whether there are applications other than the problem of motion whose explanations involve GW energy. (Germane phenomena might include the socalled CFS instability of rotating neutron stars ${ }^{79}$, (classical) black hole thermodynamics ${ }^{80}$ or astrophysical energy extraction processes. ${ }^{81}$ )

The standard explanation relies on gravitational energy, GW energy and energy-momentum conservation, all three of which are highly controversial concepts within a consistent GR framework. Here, I shall refrain from the strong claim that such concepts are illegitimate within GR. Rather, I shall grant that postulating all the quantities and assumptions the standard explanation utilizes is, at least on a practical level, unproblematic.

For a fair comparison, I first need to re-formulate and refine the standard explanation for orbital decay by explicating the more fundamental principles on which its individual steps rest. Those comprise:

- A time-varying quadrupole momentum distribution of matter (QUA), as in the dynamical explanation

- the Einstein Equations (EEs)

\footnotetext{
${ }^{79}$ Cf. Ricci/Schutz (2010), sect. 6.2

${ }^{80}$ Cf. Curiel (2014); Dougherty/Callender (2016)

${ }^{81}$ E.g. Geroch (1973)
} 
- the split (" $\Delta$ ") of the EoMs into the conservative parts (cEoMs), associated with the dynamics of the system, and non-conservative parts, associated with the dissipative gravitational radiation

From these fundamental building blocks, all three assumptions (1)-(3) of the standard interpretation can be re-defined: A system's total energy-momentum is defined as the energy associated with the conservative dynamics encoded by cEoMs; by construction, it is conserved, unless the system radiates - as previously stated in (1) and (2). Since the effective full EoMs also contain non-conservative dynamics (in other words: as a result of the cEoMsEoMs split $\Delta$ ), the GW depletes the system's energy -as previously stated in (3). The existence of non-conservative EoMs is warranted by the EEs, which also ensure the generation of GWs for a time-varying quadrupole moment of matter energy-momentum (QUA).

On the now refined standard explanation, the binary system's orbits decay, because the system's quadrupole moment varies in time (QUA), which according to the EEs triggers gravitational radiation; the difference $\Delta$ between the full EoMs and the cEoMs implies that the GWs deplete the system's total energy, manifesting itself via the effective full EoMs in orbital decay.

The refined standard explanation takes the following logical form.

$$
(\mathrm{QUA}) \stackrel{(E E s)}{\longrightarrow} \mathrm{GW} \text { emission } \stackrel{\Delta}{\rightarrow} \text { GWs deplete the system's energy } \stackrel{(\text { EoMs })}{\longrightarrow} \mathcal{E}
$$

We can now embark on a comparison between the (refined) standard explanation and the dynamical one. For the reader's benefit, I list the latter again:

$$
(\mathrm{QUA}) \stackrel{(E o M) \&(E E s)}{\longrightarrow} \mathcal{E}
$$

I first want to rebut three claims regarding putative advantages of the standard explanation its intuitive appeal and heuristic value, its close ties to observable quantities and its causalmechanistic character.

With its appeal to familiar concepts and principles, the standard explanation of the binary problem undeniably has intuitive attraction and - especially in light of the analogy with electromagnetism- heuristic value. ${ }^{82}$ Yet, many will dismiss such subjectively perceived advantages as irrelevant to an explanation's quality: Does the (putative) un-intuitiveness of quantum mechanical explanations, say, of $\alpha$-decay in terms of quantum tunneling lessen their value? Furthermore, our "intuitions" and "heuristic value" are likely to be shaped considerably by the formal approach to the Problem of Motion we adopt: The to-dated preponderant socalled " $T$-approach" (Lehmkuhl), which focuses on the energy-momentum tensor, certainly invites intuitions involving gravitational and GWs' energy-momentum. By contrast, the neglected so-called "vacuum approach", which focuses on the I.h.s. of the Einstein Equations,

\footnotetext{
${ }^{82}$ The emphasis of such analogues between GR and non-GR physical theories played a vital sociological part in GR's reinvigoration and re-integration into mainstream physics in the 1950s and 60s, cf. Schutz (2012).
} 
is no less suitable for the Problem of Motion: ${ }^{83}$ With its different outlook, explanations involving gravitational or GW energy (or energy simpliciter) seem less natural.

A different line of defence of the standard view might be this: Does the use of gravitational and GW energy perhaps enable us to ascertain more easily GR's empirical content? I fail to see how: If on the one hand one construes the argument as emphasizing the intuitive accessibility of the explanatory steps, it seems to collapse onto the already rejected appeal to familiarity. If on the other hand, one reads the argument as the claim that the standard explanation renders the observational content more explicit, it seems false: Information about the observables, i.e. the orbital decay, is no less present in the dynamical interpretation.

Perhaps most promising might be the idea that the standard explanation furnishes a causalmechanistic explanation. The guiding thought here is that energy transfer is the hallmark of causal processes ${ }^{84}$, and that causal-mechanistic explanations are pre-eminent types of explanations. But this maneuver faces two objections: Firstly, the notion of causality -and $a$ fortiori causal explanations- is notoriously ambiguous - the difficulties being even exacerbated in GR. ${ }^{85}$ Furthermore, it is not clear that causal explanations necessarily involve energy transfer: Are the explanations of action-at-a-distance theories, such as Bohmian Mechanics or Feynman-Wheeler absorber theory (neither of which prima facie involves energy transfer) uncontroversially non-causal? Secondly, it is not obvious that causal-mechanistic explanations are inherently superior to non-causal ones. Electron degeneracy pressure is explained in terms of the Pauli exclusion principle, i.e. a symmetry principle, rather than a causal mechanism. Why should this non-causal explanation of, say, white dwarf formation, impinge on its explanatory value?

Let us move on and see how the standard and the dynamical explanation each score on the central virtues of explanations, parsimony, scope, depth and unificatory power.

Regarding parsimony, it should first be pointed out that in terms of calculational efforts, both explanations are on a par: The question thus can only be which status to allocate to the split $\Delta$ the (refined) standard explanation involves. As I argued when criticism of premise (1), there is no sound a priori justification of distinguishing the conservative parts of the EoMs: After all, $\mathrm{GR}$ is supposed to supersede these less fundamental theories. In consequence, the dynamical explanation, dispensing with this distinguished treatment of the (cEoMs) i.e. the split $\Delta$, is preferable.

( $A$ fortiori, in order for the initial standard explanation to get off the ground it requires that gravitational energy and GW energy be formally definable and separable from each other which is non-trivial nor in fact always possible.)

It also deserves reiterating that the refined formulation of the standard explanation renders it transparent that claim (3), purporting the transport of GW energy-momentum, is explanatorily redundant. An inference to the best explanation for energy-momentum transport via GWs is thus blocked. Rather than contributing to the explanation of the orbital

\footnotetext{
${ }^{83}$ Cf. Lehmkuhl $(2017 a, b)$

${ }^{84}$ E.g. Dowe (2009)

${ }^{85}$ Cf. Curiel (2011)
} 
decay, it requires yet another principle to hold, viz. conservation of energy-momentum (EC), i.e. a realist interpretation of the formal energy-momentum balance of the Landau-Lifshitz type, purporting that the energy-momentum dissipated from a system must continue to exist elsewhere:

$$
(\mathrm{QUA}) \stackrel{(E E q)}{\longrightarrow} \mathrm{GW} \text { emission } \stackrel{(E o M s)}{\longrightarrow} \mathrm{GW} \text { deplete the system's senergy } \stackrel{(E C)}{\longrightarrow}(3) .
$$

Hence, if one wants to appeal to the standard explanation to abduct the GW energymomentum transport, one must first substantiate not only the privileged status of the cEoMs, but also (EC) (a realist interpretation of the energy-momentum continuity equation): This need for two additional principles then, however, seems to undermine the prerequisite for the abduction that it be the best explanation.

In summary, the verdict on parsimony disfavors the standard explanation: Why employ more explanantia than necessary (and thereby being ontologically committed to them contravening Occam's plea for parsimony)?

What about scope? Is the standard explanation able to cover a wider domain than the dynamical one? This can be negated: In higher perturbative orders, the gravitational and radiative degrees of freedom of the metric mix; it is no longer possible to systematically classify the higher order contributions of the EoMs as either pertaining to the GW or the system $^{86}$-as the standard interpretation via its split $\Delta$ presupposes. One could, of course, still insist that only the cEoMs describe the system qua their conservativeness. But such a stipulation by fiat would lack any physical foundation, for in the higher-order EoMs the radiative and the gravitational degrees of freedom cannot be severed. In short, the scope of the standard explanation is limited to low levels of approximation (viz. 2.5 or 3PPN terms). ${ }^{87}$ By contrast, the scope of the dynamical explanation coincides with the scope of GR and the general-relativistic EoMs: Wherever classical GR is valid, a dynamical explanation of a phenomenon is possible.

This leads us to the issue of depth: At what level of description do the two explanations operate? From the aforesaid, it is clear that the (refined) standard explanation with its reliance on the cEoMs employs a non-fundamental principle. By contrast, the dynamical explanation, only draws on the EoMs and the Einstein Equations, which for non-quantum purposes may without impunity be regarded as fundamental. Hence, also w.r.t. depth, the dynamical explanation prevails over the standard one. (I am not claiming that depth, thus construed, is a value per se: Sometimes, it can be traded-off for other explanatory benefits. ${ }^{88}$ Ceteris paribus, however, it seems plausible to give preference to deep explanations.)

Let us conclude our comparison with investigating the unificatory power of both explanations. Prima facie, one might be inclined to extoll the standard explanation's insistence on

\footnotetext{
${ }^{86}$ E.g. Maggiore (2007), p. 249, fn 17

87 "In my opinion, a Lagrangian or a Hamiltonian for this problem only makes sense up to 2PN order, where energy is truly conserved (if you artificially turn off the 2.5PN radiation reaction terms, you can also write down a Lagrangian for the 3PN terms). Beyond this order, the fundamental things are the equations of motion." Clifford Will, personal communication.

${ }^{88}$ E.g. Knox (2016, 2017); Franklin/Knox (2017, ms)
} 
conservative dynamics as an advantage: It thus appears to preserve continuity with the dynamics of non-GR theories. So, one might think that the standard interpretation's procedure to privilege the cEoMs (and construct from them the system's energy) can be subsumed under a successful general explanatory scheme.

The contrary, however, is the case: Firstly, as I argued, dividing the EoMs into a conservative and a non-conservative part is not even consistently feasible in GR. Such a division of the EoMs would be artificial - in contrast to pre-GR theories. Because of its reliance on this division, the standard explanation therefore cannot be subsumed under a more general explanatory scheme. In other words, the general explanatory scheme we find also in other theories is just to take the EoMs -be they conservative or not- and predict from them how matter would behave under certain conditions (MAT). This is precisely the rationale of the dynamical explanation. The latter thus can be subsumed under the widespread unificatory explanatory scheme:

$$
\text { (MAT) } \stackrel{(\text { EoMs })}{\longrightarrow} \text { explanandum. }
$$

Also regarding unification, the dynamical explanation thus seems to trump the standard one: The dynamical explanation turns out to instantiate a conservative explanatory scheme of great unificatory power, whereas the standard one unduly generalizes contingent features of preGR theories.

In summary, on the four central virtues of good explanations, parsimony, depth, scope and unificatory power, the dynamical view seems to outperform the standard one. We may thus conclude that also on purely epistemological grounds the former has much to commend itself.

The conclusion of this section is that there exists an attractive alternative to the standard explanation of the binary system's orbital decay that does not invoke gravitational or GW energy, only availing itself of the Einstein Equations and the general-relativistic Equations of Motion. The section's title question can therefore be answered in the negative. In fact, the standard interpretation should be rejected.

\section{Conclusion and outlook}

I argued that all four standard arguments for the energy-momentum transport of GWs are problematic:

(1) That a GW sets in apparent motion a ring of test masses is explained in terms of the non-Minkowskian inertial structure of spacetimes with GWs: The latter manifest themselves as deviations from the rectilinear geodesics of Minkowksi spacetime; only adopting a non-inertial frame does create the appearance of actual increase in kinetic energy.

(2) The received assumption that GWs can cause the release of surplus thermal energy in extended matter is, albeit plausible, not beyond doubt. Should it indeed be true, the 
increase in total energy can be accounted for in terms of GR's revision of energymomentum conservation for matter, which in generic spacetimes must be jettisoned. Contrariwise, the Sticky Bead Argument crucially hinges on energy-momentum conservation, which is simply assumed, to infer that GWs transmit some of their energy to the Stick Bead setup.

(3) The perturbative approach expands the metric in terms of small perturbations around a slowly varying background. These perturbations are then sourced by a term that it tempting to identify as the GW energy-momentum. While it would be folly to baulk at the use of perturbation theory on the grounds of it being not fundamental, the interpretation of the aforesaid perturbatively defined object as "GW energymomentum" rests on assumptions in conflict with GR's principles.

(4) The canonical Noether approach to GW energy yielded prima facie auspicious formal results, involving the gravitational energy-momentum pseudotensor and its associated integral quantity; but their interpretation turned out to pose essential difficulties.

We then turned to the most prominent application of GW energy, the spin-up of binary systems: According to the standard view, because the GWs carry away energy from the system, the pulsars precipitate onto more narrow orbits. I argued that such an explanation hinges on an unwarranted, and in fact not consistently feasible split of the Equations of Motion into one part, describing a conservative system, and one describing the radiation damping. A dynamical explanation, by contrast, accepts the in-spiralling orbits as a brute-fact of the full general-relativistic Equations of Motion, if the quadrupole momentum distribution of energymomentum varies in time. Furthermore, greater parsimony, scope, depth and unificatory power distinguish the dynamical explanation over the standard one. I concluded that binary systems do indeed not provide evidence for the energy-momentum transfer of GWs.

For the line of thought pursued in this paper, two directions of further enquiry are specially promising:

One concerns the more general question of the status and role of the various proposals for gravitational energy-momentum in GR. ${ }^{89}$ Of particular interest here for the philosophy and foundations of GWs, is the status of Bondi's News Function, which according to its inventor himself, "nobody has fully understood". 90

The other concerns an analysis of the role GW energy plays in relativistic astrophysics, in particular astrophysical processes during which energy is extracted from a gravitating source, such as the energy gain of a particle passing through the ergosphere of a Kerr Black Hole.

\footnotetext{
${ }^{89}$ Dürr (2017ab, ms), partly in response to Read (2017, forth.)

${ }^{90}$ Kennefick (2007), p. 208
} 


\section{Bibliography}

Aldrovandi, R. et al. (2007): "The nonlinear Essence of Gravitational Waves", in: Found.Phys. 37:1503-1517, 2007

Anderson W./Creighton. J. (2011): "Gravitational Wave Physics and Astronomy: An Introduction to Theory, Experiment and Data Analysis", Wiley

Bergmann, P. (1958): Conservation laws in general relativity as the generators of coordinate transformations" in: Phys.Rev., 112:287, 1958

Brading, K. (2005): “A Note on General Relativity, Conservation Laws, and Noether's Theorems", in: A.J. Kox/J.Eisenstaedt (eds.): "The Universe of General Relativity (Einstein Studies, Vol. 11)", Birkhäuser, 2005

Brading, K./Brown, H. (2000): "Noether's Theorems and Gauge Symmetries", http://arxiv.org/abs/hep-th/0009058

Brown, H./Brading, K. (2002): “General Covariance from the Perspective of Noether's Theorems", Diálogos 79: 59-86 (2002)

Bunge, M. (2017): “Gravitational Waves and Spacetime", in Found.Sci. (2017)

Carroll, S. (2010): http://www.preposterousuniverse.com/blog/2010/02/22/energy-is-notconserved/

Castelvecchi, D./Witze, A. (2016): "Einstein's Gravitational Waves found at last", in Nature: February/11/2016: http://www.nature.com/news/einstein-s-gravitational-waves-found-at$\underline{\text { last-1.19361 }}$

Cooperstock, F./ Tieu, S. (2012): "Einstein's Relativity: The Ultimate Key to the Cosmos", Springer

Curiel, E. (2011): "The constraints general relativity poses on physicalist accounts of causality", http://strangebeautiful.com/papers/curiel-gr-constraints-phys-caus.pdf

Curiel, E. (2014): "Black Holes are Hot”, https://arxiv.org/abs/1408.3691

Deser, S. (1970): "Self-interaction and gauge invariance", http://arxiv.org/abs/gr-qc/0411023

Dewar, N./Weatherall, J. (2017): “On Gravitational Energy in Newtonian Theories", http://philsci-archive.pitt.edu/13177/

Dirac, P. (1975): “General Theory of Relativity”, Princeton University Press

Dougherty, J./Callender, C. (2016): "Black Hole Thermodynamics: More than An Analogy?", http://philsci-archive.pitt.edu/13195/1/bht.pdf

Dowe, Ph. (2009): “Causal Process Theories" in: H. Beebee et al. (eds.): “The Oxford Handbook of Causation", Oxford University Press, 2009 
Dürr, P. (2017a, ms): “Fantastic Beasts and where (not) to Find them - Gravitational Energy in General Relativity, Part I: Local Notions", unpublished manuscript, University of Oxford

Dürr, P. (2017b, ms): "Fantastic Beasts and where (not) to Find them: Gravitational Energy in General Relativity: Part II: Global Notions”, unpublished manuscript, University of Oxford

Eddington, S.A. (1923): "The Mathematical Theory of Relativity", Cambridge University Press

Feynman, R. et al. (2002): “Feynman Lectures on Gravitation”, Westview Press

Franklin, A./Knox, E. (2017, ms): „Emergence without Limits: The case for phonons”, http://www.eleanorknox.com/uploads/3/9/8/2/39826525/phonons.pdf

Geroch, R. (1973): “Energy extraction”, in: Annals of the New York Academy of Sciences 224, 108-117. Proceedings of the Sixth Texas Symposium on Relativistic Astrophysics

Geroch, R. (2013): “General Relativity: 1972 Lecture Notes”, Minkowski Institute Press

Flanagan, E.E./ Hughes, S.A. (2005): "The basics of gravitational wave theory", http://arxiv.org/abs/gr-qc/0501041

Hobson, M.P. et al. (2006): "General Relativity: An Introduction for Physicists", Cambridge University Press

Hoefer, C. (2000): “Energy Conservation in GTR", in: Stud. Hist. Phil. Sci. B, 14 (3): 237 - 256

Kennefick, D. (2007): “Travelling at the Speed of Thought: Einstein and the Quest for Gravitational Waves", Princeton University Press

Komar, A. (1958): “Covariant conservation laws in general relativity”, in: Phys.Rev. 113: 934, 1959

Knox, E. (2016): “Abstraction and its Limits: Finding Novel Space for Explanation”, in: Nous

Knox, E. (2017): "Novel Explanation in the Special Sciences: Lessons from Physics", http://www.eleanorknox.com/uploads/3/9/8/2/39826525/novel explanation in the speci al sciences final.pdf

Lam, V. (2011): "Gravitational and Nongravitational Energy: The Need for Background Structures" in: Phil.Sci., Vol 78, No 5, 2011

Landau, L.D./Lifshitz, E.M. (1976): “Mechanics”, Butterworth-Heinemann

Landau, L.D./Lifshitz, E.M. (1971): “The Classical Theory of Fields", Butterworth-Heinemann

Leclerc, M. (2006): “Canonical and gravitational energy-stress tensors”, in: Int.J.Mod.Phys. D15 (2006)

Lehmkuhl, D. (2008): “Is spacetime a gravitational field?”, in: D. Dieks (ed.): “The ontology of spacetime, Vol II", Elsevier, 2008

Lehmkuhl, D. (2017a): "Literal vs. Careful interpretations of scientific theories: the vacuum approach to the problem of motion in general relativity", http://philsciarchive.pitt.edu/12461/ 
Lehmkuhl, D. (2017b): “General Relativity as a Hybrid Theory: The Genesis of Einstein's Work on the Problem of Motion", forthcoming in: Hist.Phil.Phys.

Lyle, S. (2008): "Uniformly accelerated charged particles: A threat to the Equivalence Principle", Springer

Maggiore, M. (2008): "Gravitational Waves - Volume 1: Theory and Experiment", Oxford University Press

Malament, D. (2012): "Topics in the Foundations of General Relativity and Newtonian Gravitational Theory", Chicago University Press

Misner, Ch. et al. (1973): “Gravitation", Freeman and Company

Mitteltaedt, P. (1981): "Philosophische Probleme der Modernen Physik" , B.I. Hochschultaschenbücher

Norton, J. (2012): "What can we Learn about the Ontology of Space and Time from the Theory of Relativity?", http://philsci-archive.pitt.edu/9113/1/Ontology 2012.pdf

Ohanian, H./Ruffini, R. (2013): “Gravitation and Spacetime”, Cambridge University Press

Ohanian, H. (2013): "The Energy-Momentum Tensor in General Relativity, and Alternative Theories of Gravitation, and the Gravitational vs. Inertial Mass", http://arxiv.org/abs/1010.5557

Padmanabhan, T. (2004): "From Gravitons to Gravity: Myths and Reality", Int.J.Mod.Phys.D17:367-398, 2008

Padmanabhan, T. (2010): "Gravitation: Foundations and Frontiers", Cambridge University Press

Papapetrou, A. (1957): “Über periodische nicht-singuläre Lösungen in der allgemeinen Relativitätstheorie", in: Ann. Phys. 20, 399, 1957

Papapetrou, A. (1958): "Über zeitabhängige Lösungen der allgemeinen Relativitätstheorie", in: Ann. Phys. 1, 185, 1958

Peskin, M./Schroeder, D. (1995): “An Introduction to Quantum Field Theory”, Westview Press

Petkov, V. (2012): “Inertia and Gravitation: From Aristotle's Natural Motion to Geodesic Worldlines in Curved Spacetime", Minkowski Institute Press

Petkov, V. (2017): “Is Gravitation Interaction or just curved Spacetime Geometry?", http://philsci-archive.pitt.edu/12722/1/Gravitation.pdf

Pitts, B. (2016a): “Einstein's Physical Strategy, Energy Conservation, Symmetries, and Stability: 'but Grossmann \& I believed that the conservation laws were not satisfied'", in: Stud. Hist. Phil.Mod.Phys. 54 (2016) pp. 52-72

Pitts, B. (2016b): “Einstein's Equation for Spin 2 Mass 0 from Noether's Converse Hilbertian Assertion", http://philsci-archive.pitt.edu/12464/ 
Poisson, E./Will, C. (2014): “Gravity. Newtonian, Post-Newtonian”, Cambridge University Press

Rohrlich, F. (2007): “Classical Charged Particles”, World Scientific

Read, J. (2017, ms): "Functional Gravitational Energy", forth. In: BrJPS

Rey, D. (2013): "Similarity Assessments, Spacetime, and the Gravitational Field: What Does the Metric Tensor Represent in General Relativity?", http://philsci-archive.pitt.edu/9615/

Schrödinger, E. (1950): "Space-time structure”, Cambridge University Press

Schutz, B. (2012): "Thoughts about a conceptual framework for relativistic gravity" in C. Lehner et al. (eds.): "Einstein and the Changing Worldviews of Physics", Birkhäuser, 2012

Schutz, B./Ricci, F. (2010): "Gravitational Waves, Sources, and Detectors", http://arxiv.org/abs/1005.4735

Stachel, J. (1993): "The Meaning of General Covariance: 30 Years of Dispute" in: J. Earman et. al. (eds.): "Philosophical Problems of the Internal and External Worlds", University of Pittsburgh Press

Straumann, N. (2013): “General Relativity: With Applications to Astrophysics", Springer

Trautmann, A. (1962): "Conservation Laws in General Relativity", in: L. Witten (ed.): "Gravitation: An Introduction to Current Research", John Wiley, 1962

Vollmer, G. (2010): “Invariance and Objectivity”, Found. Phys. 40 (9-10): 1651-1667 (2010)

Wallace, D. (2012): "The Emergent Multiverse: The Universe according to the Everett Interpretation", Oxford University Press

Weinberg, S. (1972): "Gravitation and Cosmology", Wiley

Weyl, H. (1922): "Raum-Zeit-Materie: Vorlesungen über Relativitätstheorie", Springer (reprint: 1993)

Will, C. (2014): "The Confrontation between General Relativity and Experiment", http://relativity.livingreviews.org/Articles/Irr-2014-4/ 